\title{
THE EFFECT OF MAPS UPON THE DIMENSION OF SUBSETS OF THE DOMAIN SPACE
}

\author{
R. F. WILLIAMS
}

"Space filling curves" constitute simple examples of maps which raise the dimension of each closed subset of the domain space of positive dimension. That is, in its usual construction, a map $f$ of a 1-cell $X$ onto an $m$-cell $Y$ is such that each closed subset of positive dimension, or what amounts to the same, each nondegenerate subcontinuum of $X$, has an image of dimension $m$.

In case the dimension of $X$ is greater than 1, maps with such strong dimension raising properties do not always exist. For example, there does not exist a map of a 2-cell into a 3-cell such that the image of each nondegenerate sub-continuum is 3 , or even $\geqq 2$. This fact is contained in the results below, all of which give some bound to the dimension raising possibilities of maps.

It should be noticed that for $m>n$, there do exist maps (of the $n$-cell $I^{n}$ onto the $m$-cell $\left.I^{m}\right)$ such that the dimension of all $n$-dimensional sets are raised. Thus $f g: I^{n} \rightarrow I^{m}$ is such a map, where $g$ is the projection of $I^{n}$ onto a 1-cell $I^{1}$ and $f$ is as indicated in the first paragraph. Here, certain $(n-1)$-dimensional sets are mapped by $g$ and hence by $f g$ into points. This can be slightly improved ( $I^{i}$ as before):

EXAMPLE. There exists a map $F: I^{n} \rightarrow I^{m}$, for all $n, m>n$, such that the dimension of each $n$-dimensional set is increased by $F$ and the dimension of no closed set is decreased by $F$.

Let $f: I^{1} \rightarrow I^{m-n+1}$ be as in the first paragraph and let $f_{i}$ denote the $i$ th coordinate map of $f$, for $i=1,2, \cdots, m-n+1$. Define $F: I^{n} \rightarrow I^{m}$ by

$$
F\left(x_{1}, x_{2}, \cdots x_{n}\right)=\left(x_{1}, x_{2}, \cdots x_{n-1}, f_{1}\left(x_{n}\right), f_{2}\left(x_{n}\right), \cdots f_{m-n+1}\left(x_{n}\right)\right) .
$$

Then $F$ is light as $f$ is; light maps on compact sets cannot decrease dimension (see $[1$, p. 91 or Theorem $C$ below). The image of each $n$-dimensional set is of dimension $m$, as $F$ inherits from $f$ the property of sending open sets into sets with nonempty interiors.

Throughout, $f$ will be a map of a separable metric space $X$ of dimension $n$ into a separable metric space $Y$ of dimension $m$.

Presented to the Society, December 27, 1955 under the title Concerning dimension raising mappings of cells and on April 14, 1956 under the title $A$ note concerning dimention raising maps; received by the editors May 8, 1956 and, in revised form, September 19, 1956. 
These first four results are corollaries to Theorems A, B, and C below:

(1) If $m<2 n$, then $f$ does not raise dimension of all closed subsets of dimension $1(n \geqq 1)$.

(2) If for some positive integer $i, n=2 i$ and $m=2 i+1$, then there is a closed set of dimension $i$ whose dimension is not raised.

(3) If $f$ increases the dimension of all closed 1-dimensional sets, then $f$ must at least double the dimension of all closed sets.

(4) If $X$ is compact and $n=m$, then among those closed sets whose dimensions are not raised by $f$ are sets of all dimensions $\leqq n$.

We first prove Theorems A, B, and C and a "remark" which is of a familiar nature; $\mathrm{C}$ is fairly well known and is included for the sake of completeness.

REMARK. If $Z$ is a subset of $X$ of dimension $i$, then there is a closed set $X^{\prime} \subset X-Z$, of dimension $n-i-1$.

Proof. As $X$ is $n$-dimensional, there exist closed sets $C_{1}$ and $C_{2}$ in $X$ such that no set of dimension $\leqq n-2$ separates $C_{1}$ and $C_{2}$ and such that $C_{1} \cdot C_{2}=0$. By Theorem "B" of $[1$, p. 34], there exists a closed set $B_{1}$ separating $C_{1}$ and $C_{2}$ such that $\operatorname{dim} Z \cdot B_{1} \leqq i-1$. As $B_{1}$ separates $C_{1}$ and $C_{2}, \operatorname{dim} B_{1} \geqq n-1$. Repeating this process, we obtain a closed set $B_{2} \subset B_{1}$ such that $\operatorname{dim} Z \cdot B_{2} \leqq i-2$ and $\operatorname{dim} B_{2} \geqq n-2$, a closed set $B_{3}, \cdots, B_{i+1}$. Then $\operatorname{dim} Z \cdot B_{i+1} \leqq-1$ and $\operatorname{dim} B_{i+1} \geqq n-i-1$, so that $B_{i+1}$ is the required closed set $X^{\prime}$.

Theorem A. There is a closed subset of $X$ of dimension at least $(n-1) / 2$ whose image is of dimension at most $m / 2$.

Proof. This is clear if $m=0$. If $m>0$, let $p$ and $q$ denote non-negative integers such that $p \leqq m / 2, q \leqq m / 2$, and $p+q=m-1$. By the corollary to Theorem III 3, [1, p. 32], $Y$ can be expressed as the sum of two sets, $P$ of dimension $p$, and $Q$ of dimension $q$. Though not noted in [1], the proof given shows that $P$ can be taken to be an $F_{\sigma}$ set. Then $f^{-1}(P)$ is an $F_{\sigma}$ set, say $f^{-1}(P)=\bigcup_{i=1}^{\infty} F_{i}$, where each $F_{i}$ is closed.

Case 1 . For some $i, \operatorname{dim} F_{i} \geqq(n-1) / 2$. Then $F_{i}$ is a closed set whose image is of dimension $\leqq \operatorname{dim} P \leqq m / 2$.

Case 2. For all $i, \operatorname{dim} F_{i}<(n-1) / 2$. Then $\operatorname{dim} f^{-1}(P)<(n-1) / 2$ (Sum Theorem, $[1$, p. 30]). Then by the remark above, there is a closed set $X^{\prime} \subset X-f^{-1}(P)=f^{-1}(Q)$, of dimension $\geqq n-(n-1) / 2-1$ $=(n-1) / 2$. As the image of $X^{\prime}$ lies in $Q$, it has dimension $\leqq m / 2$.

Theorem B. Either there is a closed 1-dimensional set in $X$ whose image is at most 1-dimensional, or there is a closed ( $n-1)$-dimensional set in $X$ whose image is at most $(m-2)$-dimensional. 
Proof. Let $Y=P+Q$, where $P$ is a 1 -dimensional $F_{\sigma}$ set, $Q$ is $(m-2)$-dimensional, and $P \cdot Q=0$. As in the proof of Theorem A, if $\operatorname{dim} f^{-1}(P) \geqq 1$, then $f^{-1}(P)$ contains a 1 -dimensional closed set whose image, lying in $P$, is at most 1 -dimensional. If $\operatorname{dim} f^{-1}(P)<1$, that is, $\leqq 0$, then $f^{-1}(Q)$ contains a closed set of dimension $\geqq n-1$, whose image, lying in $Q$, has dimension $\leqq m-2$.

THEOREM C. If $X$ is compact, then there is a closed set of dimension $\geqq n-1$ whose image is of dimension $\leqq m-1$.

Proof. $X$ contains an $n$-dimensional Cantor manifold $X^{\prime}$. Let $g=f \mid X^{\prime}$. There exists a compact set $Q \subset g\left(X^{\prime}\right)$ of dimension $\leqq m-1$ which separates $g\left(X^{\prime}\right)$. But then $g^{-1}(Q)$ separates $X^{\prime}$ and is therefore of dimension $\geqq n-1 ; f^{-1}(Q)$ is closed and has an image of dimension $\leqq m-1$.

Proof of Corollaries 1-4. Theorem B implies (1) by induction. Theorem A applied to (2) yields a closed set $M$ such that $\operatorname{dim} M$ $\geqq(2 i-1) / 2$ and $\operatorname{dim} f(M) \leqq(2 i+1) / 2$. As these dimensions have to be integers, $\operatorname{dim} M \geqq i \geqq \operatorname{dim} f(M)$. Corollary 3 follows from (1). Theorem $\mathrm{C}$ implies (4) by induction.

It is to be noticed that Theorems A, B, and C give certain information about the closed subsets of $X$ of intermediate dimension:

(5) If for some $i, n \geqq 2^{i}$ and $m<2^{i+1}$, then among those closed sets whose dimensions are less than doubled by $f$, are sets of dimension $1,2,4, \cdots, 2^{i}$.

(6) If for some integers $i$ and $j, i \geqq j, n \geqq 2^{i}$, and $m<2^{i}+2^{j}$, then there exists a closed set of dimension $2^{i-j}$ whose dimension is not raised.

(7) If $X$ is compact and for some integers $i$ and $j, i \geqq j, n \geqq 2^{i}$, and $m<2^{i}+2^{i}$, then among those continua in $X$ whose dimensions are not raised, are continua of all dimensions $\leqq 2^{i-i}$.

Proof of Corollaries 5-7. Corollary (5) is obtained by applying Theorem A, $i$ times, together with the fact that a closed set of dimension $\geqq k$ contains a closed set of dimension $=k$. Similarly, Theorem A applied $j$ times yields (6); (7) follows from (6) and Theorem C.

Corollaries (1), (2), (4), (6), and (7) may be considered as attempts to determine the largest integer $k=k(n, m)$ such that:

If $f: X \rightarrow Y$, then among those closed sets whose dimensions are not increased by $f$ are sets of all dimensions $\leqq k(n, m)$.

A tabulation of $k(n, m)$ follows, where the occurrence of " $i$ ?" means that the corresponding value of $k$ is known only to be at least $i$.

Perhaps the most interesting portion of the problem of determining the integers $k(n, m)$ which remains, can be phrased as follows:

QUESTION. Is there a map $f$ defined on a space $X$ of dimension 


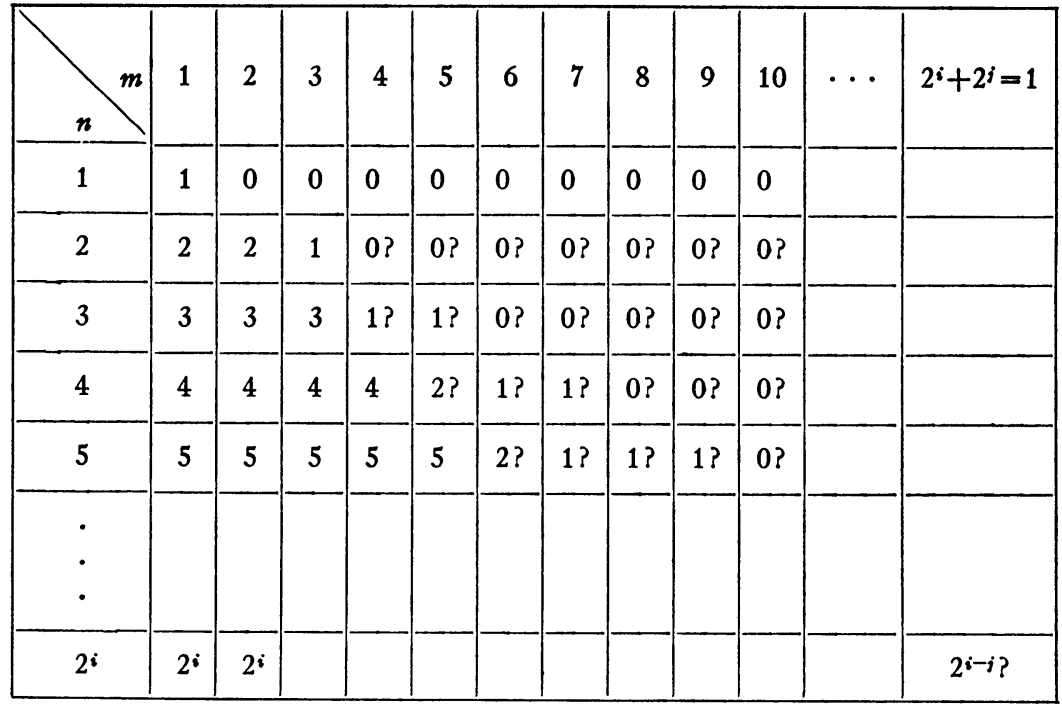

$n \geqq 2$ which raises the dimension of each closed subset of $X$ of positive dimension? Could $f$ be a map of $I^{2}$ into $I^{4}$ ?

If there is such a map, Corollary (3) shows that it is sufficient to consider only the 1-dimensional sets and hence, for compact $X$, only the nondegenerate continua.

\section{BIBLIOGRAPHY}

1. W. Hurewicz and H. Wallman, Dimension theory, Princeton, 1948.

UNIVERSITY OF WISCONSIN

\section{COMMENTS ON OPEN HOMOMORPHISMS ${ }^{1}$}

\section{B. J. PETtis}

The present note consists of two comments on the openness of certain homomorphisms, between linear topological spaces (1.t.s.) in the first case and metric groups in the second. Both comments present only refinements on earlier results; the methods, however, are somewhat different and may be of some interest.

Let $\mathfrak{A}$ be any class of closed convex circled sets defined in every

Presented to the Society, April 20, 1951; received by the editors July 15, 1956.

1 This paper was done under Contract N7-ONR-434, Office of Naval Research. 